\title{
In-core dosimetry for the validation of neutron spectra in the CROCUS reactor
}

\author{
Y.Jiang, A.Laureau, V. Lamirand ${ }^{*}$, P. Frajtag, A.Pautz \\ Ecole Polytechnique Fédérale de Lausanne (EPFL) \\ * vincent.lamirand@epfl.ch
}

\begin{abstract}
The present article describes the preliminary validation study of simulated in-core and reflector $n$ eutron spectra in preparation of oncoming experimental programs in the zeropower reactor CROCUS at EPFL. For this purpose, a set of activation foils were irradiated at three characteristic positions in the CROCUS reactor, and the subsequent activities were analyzed via $\gamma$ spectrometry. The experimental setup was then modeled with the Monte Carlo neutron transport code Serpent2 and associated with an analysis tool to include the effect of the reactor power history during experiments.

The comparison of calculated and measured reaction rates $(\mathrm{C} / \mathrm{E})$ indicates a general consistency (at $2 \sigma$ ) between calculated and measured spectra. However, offsets of $\mathrm{C} / \mathrm{E}$ values were observed in $(n, \gamma)$ reactions, up to $18 \%$ for ${ }^{115} \mathrm{In}$ and $8 \%$ for ${ }^{63} \mathrm{Cu}$ dosimeters. This could be caused by an unexpected isotopic composition, uncertainties in nuclear data, or the spectrometry analysis.

In addition, a 100-groups spectrum unfolding was performed using the experimentally determined reaction rates and the Serpent 2 spectra as the prior knowledge. The unfolded spectra were mainly adjusted in the thermal and fast ranges, while few modifications $w$ ere $m$ ade i $n t$ he e pithermal $r$ egion $d$ ue $t o$ the low contribution of epithermal neutrons in activation processes. Moreover, within energy groups where the capture reactions show resonant behavior, flux depletion (up to $38 \%$ as compared to the prior spectra) is observed due to the absence of self-shielding effect in the unfolding process. For this purpose, an unfolding method based on energy groups weighting is developed and tested.
\end{abstract}

Keywords-Neutron spectrum measurement, in-core dosimetry, gamma spectrometry, spectrum unfolding

\section{INTRODUCTION}

The development of the knowledge on nuclear data have largely contributed to the advances of reactor technology, while continuous improvement is still required for further optimization, new designs and waste management for example.

The nuclear data of stainless steel components ( $\mathrm{Fe}, \mathrm{Cr}$ and $\mathrm{Ni}$ ) are of great interest for the performance assessment of the Gen-III EPR reactor. Validation of the iron nuclear data was accomplished in the EOLE reactor for this purpose [1]. In continuation of this work, the PETALE program [2]-[4] will be performed in the zero-power reactor CROCUS. For such

All authors are with the Laboratory for Reactor Physics and Systems behaviour, Ecole Polytechnique Fédérale de Lausanne, CH-1015 Lausanne, Switzerland. Y.Jiang is the corresponding author, currently with the Experimental Physics Service, French Atomic Energy Commission, 13108 SaintPaul-lez-Durance, France (e-mail: yifeng.jiang@cea.fr).

V.Lamirand is with the Laboratory for Reactor Physics and Thermal Hydraulics, Paul Scherrer Institut, CH-5232 Villigen, Switzerland.

A.Pautz is with the Nuclear Energy and Safety Research Division, Paul Scherrer Institut, CH-5232 Villigen, Switzerland. program, the interpretation of experimental data would require a precise knowledge of neutron energy spectra.

In this paper is described the experimental study conducted in CROCUS, for the validation of simulated in-core and reflector neutron spectra by means of activation dosimetry.

\section{METHOD}

\section{A. Experimental setup}

CROCUS is a an experimental reactor operating at a maximal power of $100 \mathrm{~W}$. The reactor is light water-moderated with two interlocked fuel zones in a square lattice, separated by a water gap, as shown in fig. 1 . The power of the reactor is monitored by two ex-core fission chambers and two $\gamma$ compensated ionization chambers. Further information on the
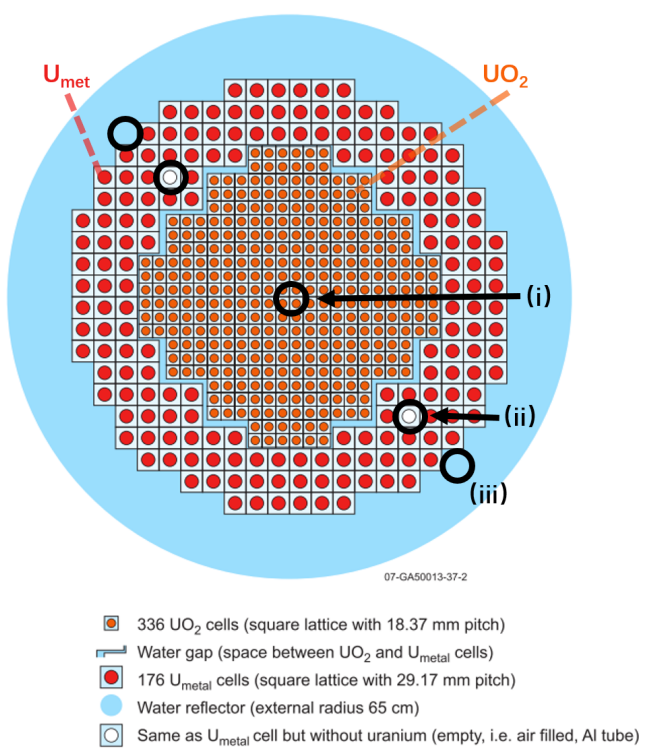

Figure 1: CROCUS core configuration with irradiation positions for the present study (after [5])

reference experimental configuration of the reactor can be found in [5].

For the current study, disk-form dosimeters are placed at three different positions (indicated in fig. 1) within the reactor: (i) at the center of the core, (ii) in one of the empty control rod tubes, (iii) in two empty aluminium rods placed in the near periphery of the core (surrounded by the water reflector). Dosimeters are prepared and laminated in a thin plastic film to avoid possible deformation and contamination during experiments. These dosimeters are then attached to Plexiglas plates for positioning. 
At the end of the experiments, safety systems are activated to shut down the reactor. The activation of dosimeters are then analyzed via $\gamma$ spectrometry, using High Purity Germanium (HPGe) detectors [6], [7].

\section{B. Experimental design}

The experimental design consists of selecting dosimeters, reactor power and operation time. It was accomplished with simulated activation experiments using the Monte Carlo neutron transport code Serpent2 [8], under criticality configuration based on the modeling in [9].

For illustration purpose, some simulated flux profiles (in 100 energy groups) at different experimental positions are shown in fig. 2, while the shaded areas represent the $1 \sigma$ uncertainty of the simulation. The calculation is made in a $0.005 \mathrm{~cm}^{3}$ detector, which is representative of the size of dosimeters.

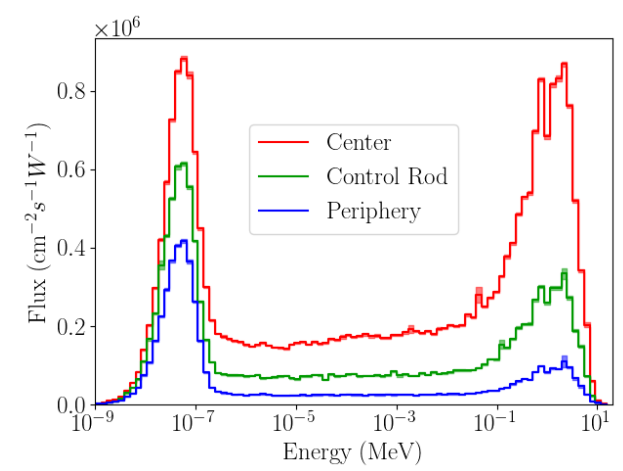

Figure 2: Simulated flux in different experimental positions

One can observe a symmetrical profile $f$ or $t$ he thermal and the fast contributions in the core center, while the fast component drops significantly outside the fuel zone. Based on the flux profiles, it was decided to use two types of dosimeter: (1) resonant dosimeters presenting capture reactions $(n, \gamma)(2)$ threshold dosimeters with threshold reactions such as $(n, p)$ or $(\mathrm{n}, \mathrm{n}$ ').

The dosimeters are selected according to the contribution of the calculated reaction rates (RR) in different energy groups between $1 \times 10^{-5} \mathrm{eV}$ and $20 \mathrm{MeV}$. Figure 3 illustrates the sensitivity of different dosimeters in terms of distributed and integral RR. Resonant dosimeters are sensitive to the thermal flux a nd c ertain $g$ roups of e nergy w here there a re resonant peaks in the cross section. On the other side, threshold dosimeters are meant to target the fast flux since the threshold energy of the reaction is generally above $0.5 \mathrm{MeV}$. Additionally, resonant dosimeters covered with neutron poison (cadmium boxing) are also used to target epithermal contribution. The set of dosimeters (reactions and associated decay data) used for spectrum characterization is listed in Table 1.

\section{Analysis with simulations}

An effort has been made to accurately reproduce the experimental configurations in simulation for comparison purpose. In the Serpent 2 calculations, dosimeters are modeled with their exact geometry and position within the reactor during

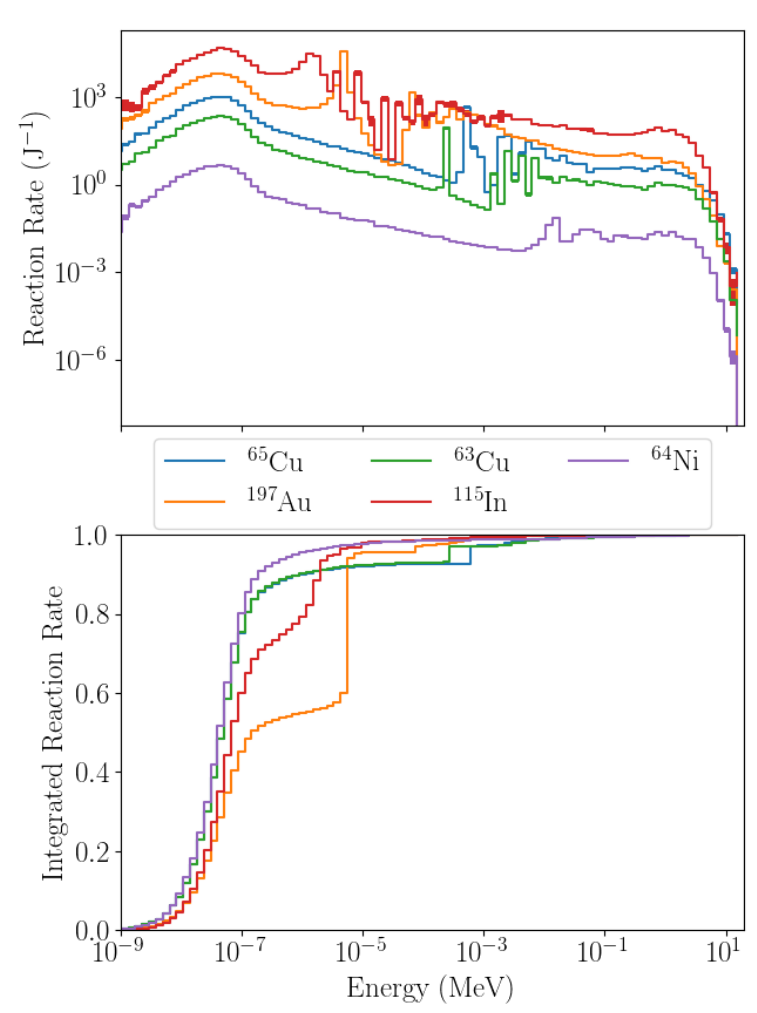

(a) Capture reactions
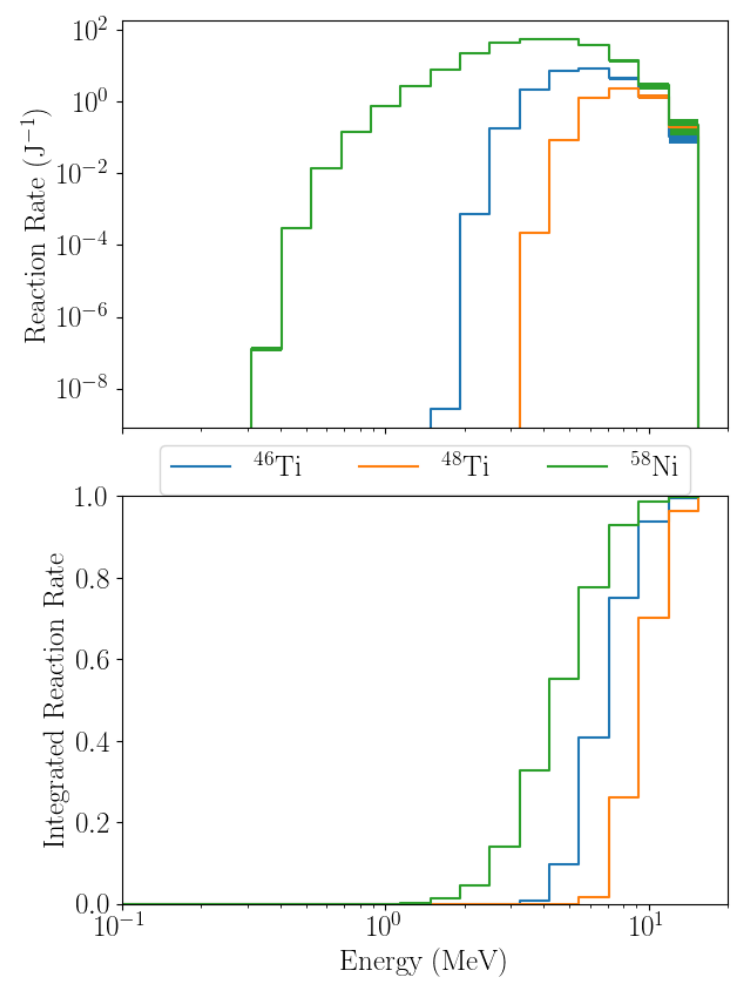

(b) Threshold reactions

Figure 3: Distribution of reaction rates for different types of dosimeters: reaction rate (top) and integrated reaction rate (bottom) for the considered capture and threshold activation reactions 
experiments. The isotopic composition of the materials are assumed to be as the natural abundance. The activity $A_{i}(t)$ for a certain isotope $i$ is estimated using the calculated RRs $R_{i}$ and the reactor power history $P(t)$.

For precise activity estimation of short-lived isotopes, the power history is of importance. Consistently, the influence of power history is checked for all isotopes of interest. This is implemented in a numerical code based on a simplified Bateman equation, by neglecting reaction rates in isotopes once activated:

$$
A_{i}\left(t_{n}\right)=\lambda_{i} \Delta t \sum_{k=0}^{n}\left[C_{0} R_{i} P\left(t_{k}\right)-A_{i}\left(t_{k}\right)\right]
$$

where $\lambda_{i}$ is the decay constant, $\Delta t$ the elementary time step and $C_{0}$ the normalization constant. This normalization is achieved with a reference ${ }^{197} \mathrm{Au}(\mathrm{n}, \gamma)$ dosimeter in each experiment.

A direct comparison can therefore be made between the measured dosimeter activity $A_{E}$ and the simulated one $A_{C}$. These quantities are proportional to the measured and calculated RRs $R_{E}$ and $R_{C}$. The ratio $\mathrm{C} / \mathrm{E}$ between $\mathrm{RRs}$ in calculation (C) and experiment (E) can be estimated as:

$$
\frac{C}{E}=\frac{A_{C}}{A_{E}}=\frac{R_{C}}{R_{E}}
$$

In addition to the validation based on $\mathrm{C} / \mathrm{E}$, the calculated neutron spectrum can be adjusted by the experimental feedback of measurements $\left(R_{E}\right)$. This is accomplished via an unfolding procedure that compares the measured RR and that for an initial guess spectrum (the one calculated in Serpent2 in the present study). The best approximated spectrum is obtained by minimizing the residual on RRs in the $\chi^{2}$ sense. The unfolding is done with a reference code MAXED [10] based on a maximum entropy criterion, and two non-linear optimization methods with the CVXPY package [11] in Python. The first method uses augmented response matrix (of cross sections) and the information of the spectrum (referred as the unweighted method hereafter). The other method uses the spectral distribution of RRs calculated in Serpent2 to weight the contribution of the response in each energy group. Thus, it will be referred as the weighted method. The unweighted method uses a response matrix of constant cross section values, therefore the self-shielding is not taken into account. The weighted method, by contrast, aims at introducing implicitly the self-shielding effect into the unfolding by assigning different importance to energy groups.

\section{RESULTS AND DISCUSSION}

In the present part, the comparison of RRs in simulations and experiments is summarized. The coherence between calculation and measurement indicates that the Serpent 2 modeling using a common purpose JEFF 3.1.1 library [12] is in general consistent with outcomes of the activation dosimetry. The result of the comparison also allowed a study on spectrum unfolding. However, discrepancies between calculated and measured RRs are observed for some dosimeters. Possible sources of these discrepancies will be discussed.

\section{A. $C / E$ in thermal and epithermal ranges}

The overview of $\mathrm{C} / \mathrm{E}$ values for resonant dosimeters, therefore sensitive to thermal and epithermal ranges, is presented in fig. 4.

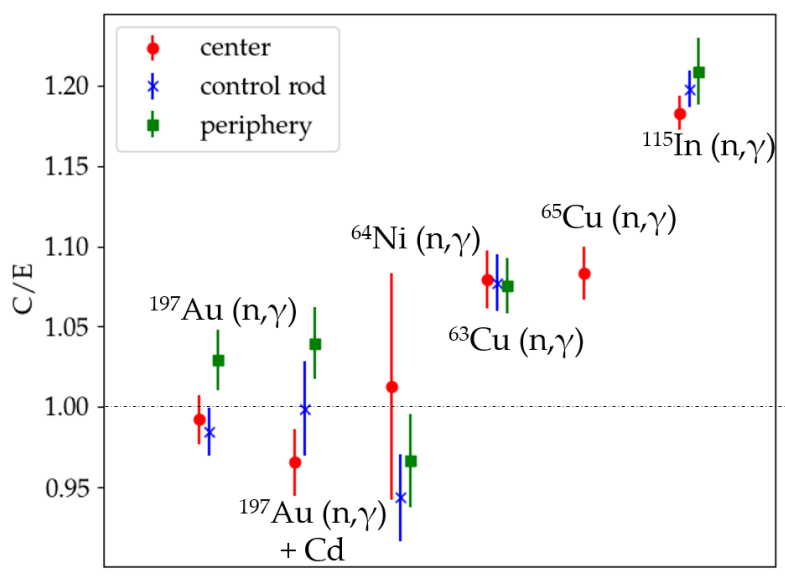

Figure 4: $\mathrm{C} / \mathrm{E}$ values for resonant dosimeters at different positions

For each reaction at the three experimental positions, one representative and consistent value throughout a number of experiments is shown with its uncertainty. The $\mathrm{C} / \mathrm{E}$ values indicate the agreement between simulations and measurements either with the absolute value or the relative one. It should be noted that uncertainties associated to resonant dosimeters are mostly small as their high activation allows a precise determination of the activity. The relatively large uncertainty for ${ }^{64} \mathrm{Ni}$ is partly due to its low isotopic proportion in nature $(<$ $1 \%)$, partly due to its short half-life (2.5 hours). Discrepancies for ${ }^{63} \mathrm{Cu},{ }^{65} \mathrm{Cu}$ and ${ }^{115} \mathrm{In}$ dosimeters were observed to be an offset, regardless of the position of the dosimeter across different experiments. For ${ }^{63} \mathrm{Cu}$ and ${ }^{65} \mathrm{Cu}$, the uncertainty in cross section data and branching ratio of $\gamma$ decay emission is of the same order of magnitude [12]-[15]. In the case of ${ }^{115}$ In the source of the offsets could be a possible isotopic composition that is different from natural abundance, or biases and uncertainties in $\gamma$ spectrometry analysis not taken into account in this study.

It is worth noticing that the activity measurement of ${ }^{63} \mathrm{Cu}$ is made on a particular $\gamma$ ray: the $511 \mathrm{keV}$ photon created through the annihilation of a positron over an electron. It is however difficult to confirm that the background annihilation in the acquisition system during measurements is fully subtracted from the target decay emissions. Therefore, the applicability of spectrometry analysis is questionable by definition. It was also observed that the acquired peak shape in $\gamma$ spectrum, illustrated in fig. 5, resembles that of a Gaussian (Poisson distribution of a large number of events), but the width of the peak is generally two times larger than ordinary peaks measured in this work.

\section{B. $C / E$ in the fast range}

Despite the general agreement between simulation and experiment for threshold dosimeters as shown in fig. 6, large 


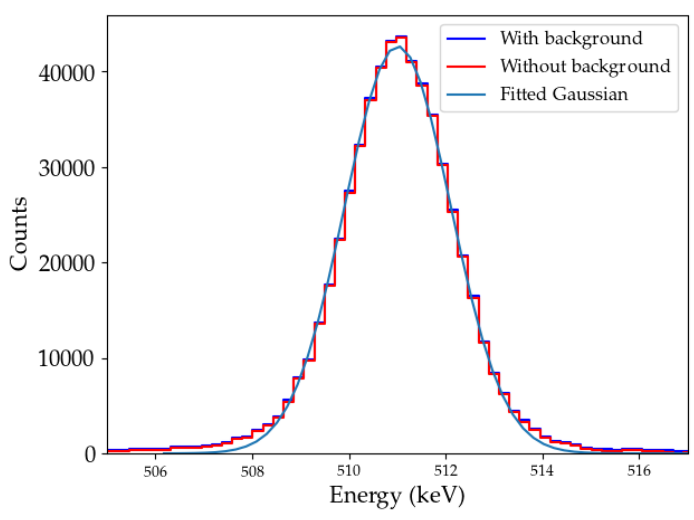

Figure 5: Illustration of measured annihilation peak of ${ }^{64} \mathrm{Cu}$ decay $\gamma$ spectrum

uncertainties were observed due to difficulties in measuring the activity and high statistical uncertainties in the RR calculations.

In addition to the dosimeters presented in fig. 6 , the inelastic scattering reaction (n,n') of ${ }^{115}$ In was also measured in experiments, through the $336 \mathrm{keV}$ photon emission during the transition from the first metastable level to its fundamental level. However, the results show important discrepancies between calculation and measurement: the calculated RRs is about $6 \%$ of the measured one. This issue was also reported by [16]. A possible explanation is that, in general purpose nuclear data libraries, inelastic scattering cross section is defined for different metastable levels while in $\gamma$ spectrometry the activity is measured as the sum of all transitions that would contribute to the metastable level of interest. The complexity of the decay scheme makes inconceivable to compare calculations with measured inelastic scattering RR through common libraries.

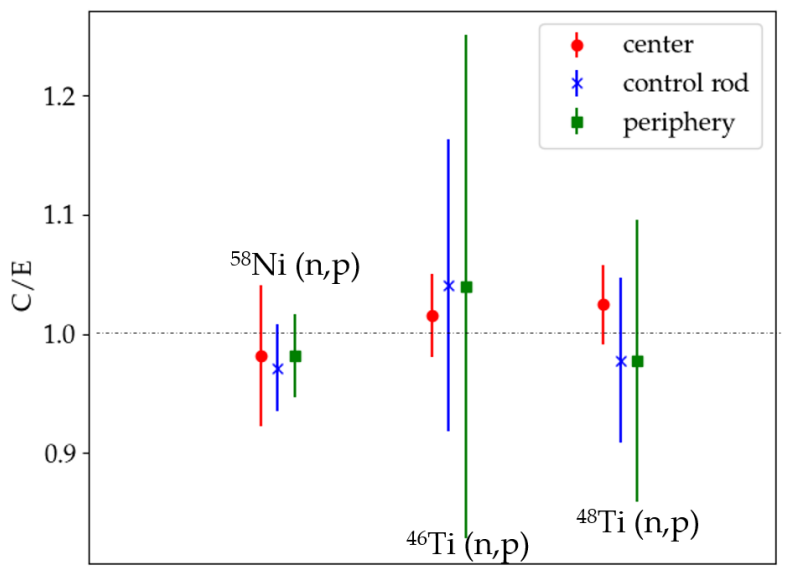

Figure 6: $\mathrm{C} / \mathrm{E}$ values for threshold dosimeters at different positions

Specialized libraries for dosimetry, for instance IRDFF, provide dosimetry cross section files, which estimate in terms of inelastic scattering cross section the overall yield of the metastable level being considered. Thus, the use of specialized libraries for $\left(n, n^{\prime}\right)$ reactions will be considered in future analysis.

\section{Spectrum unfolding}

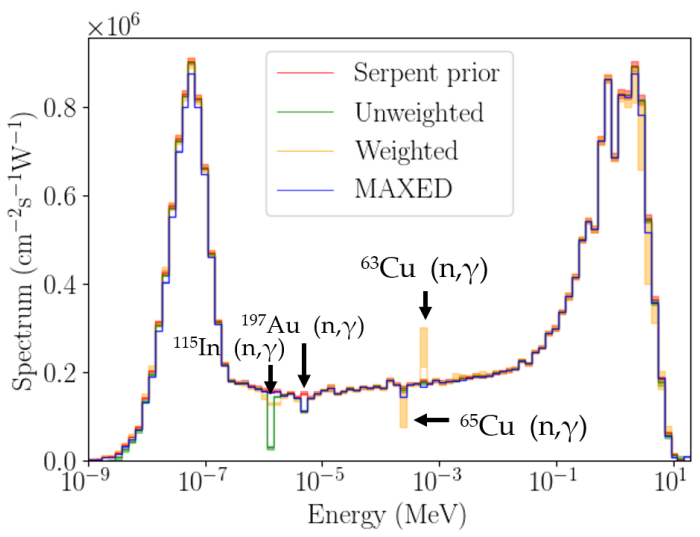

(a) Core center

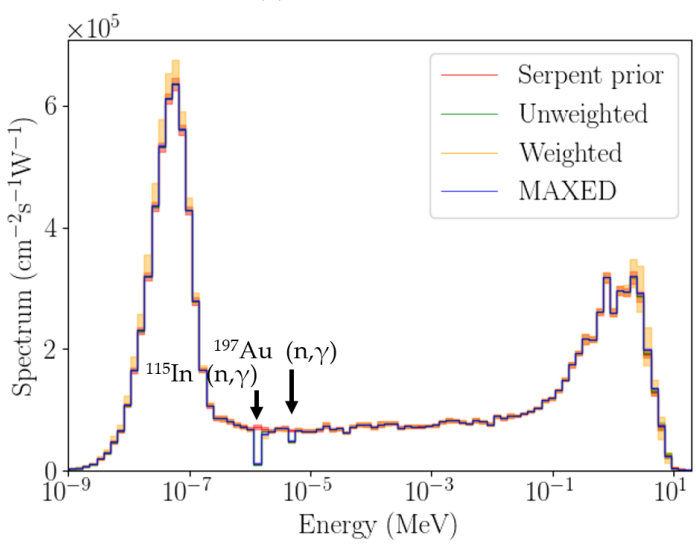

(b) Control rod

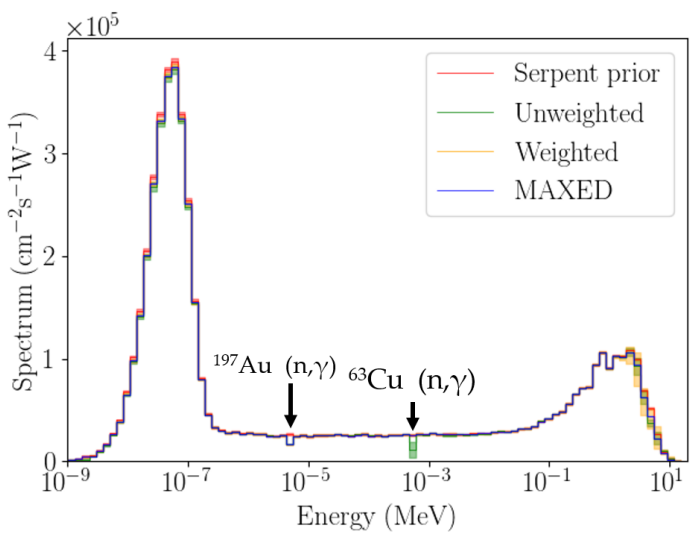

(c) Periphery

Figure 7: Unfolded neutron spectra for experimental positions

As indicated in fig. 7, the unfolding results are generally in agreement with the initial Serpent2 spectra. Sharp depletion (up to $38 \%$ as compared to the prior spectrum) or augmentation of the flux is observed in the epithermal range, which is caused by the large value of resonant capture cross sections. Energy groups with strong flux variation are identified to be associated with resonance peaks of different 
reactions (fig. 7). In reality, the self-shielding limits the actual $\mathrm{RR}$ in the resonance region, while in the unfolding this mechanism has to be integrated numerically. This situation can be illustrated through the comparison of unfolded spectra by different methods: The unweighted method takes the cross section values as constant and independent of the spectrum, therefore the unfolded spectra show serious flux variation (fig. 7a). The weighted method shows positive results for the self-shielding correction. With a relatively high cost associated to flux adjustment in resonance regions, the self-shielding effect is represented to a certain extent. Nevertheless, in the case of the spectrum at the control rod position (fig. 7b) the flux depletion is important regardless of the method, possibly caused by numerical issues. Additionally, calculated and measured uncertainties in the RR of threshold dosimeters lead to large uncertainty in the unfolded fast spectra. Further improvement on the experimental procedure and more accurate reaction rate estimation in simulation are expected to optimize the unfolding results.

\section{CONCLUSION}

This paper summarizes the used methodology to validate simulated neutron spectra in the zero-power reactor CROCUS for dosimetry applications. A set of activation dosimeters were chosen, so that the energy spectrum from thermal to up to $20 \mathrm{MeV}$ is covered as much as possible. In-core experiments were designed by Monte Carlo modeling, and performed at different locations in CROCUS and the consequent activation is measured via $\gamma$ spectrometry. The experimental setup were then accurately modeled to compare calculations with experimental results in terms of $\mathrm{C} / \mathrm{E}$. The general agreement on $\mathrm{C} / \mathrm{E}$ indicates the consistency between calculated and measured spectra, and thus the validation of spectra and experimental methodology.

Spectrum unfolding was performed using experimental feedbacks. Two unfolding methods were implemented, which show larger uncertainty in the solution spectrum, but are consistent with the MAXED reference solution.

Inconsistency and offsets in $\mathrm{C} / \mathrm{E}$ were observed in the analysis, which could be caused by different sources of biases in simulation, experiments and nuclear data. Further improvements are to be made on the design of activation experiments, along with a dedicated model for corrections in spectrometry analysis.

\section{REFERENCES}

[1] C Vaglio-Gaudard, A Santamarina, P Blaise, O Litaize, A Lyoussi, G Noguère, J. M. Ruggieri, and J. F. Vidal, "Interpretation of PERLE Experiment for the Validation of Iron Nuclear Data Using Monte Carlo Calculations," Nuclear Science and Engineering, vol. 166, no. 2, pp. 89-106, 2010.

[2] V. Lamirand, G. Perret, S. Radman, D. Siefman, M. Hursin, P. Frajtag, A. Gruel, P. Leconte, P. Blaise, and A. Pautz, "Design of separated element reflector experiments in CROCUS: PETALE," in International Symposium on Reactor Dosimetry, 2017, p. 7.
[3] A. Laureau, V. Lamirand, D. Rochman, and A. Pautz, "Total Monte Carlo acceleration for the PETALE experimental programme in the CROCUS reactor," Accepted in WONDER 2018 - 5th edition of the International Workshop On Nuclear Data Evaluation for Reactor Applications, 2018.

[4] V. Lamirand, A. Laureau, D. Rochman, G. Perret, A. Gruel, P. Leconte, P. Blaise, and A. Pautz, "An Experimental Programme optimized with Uncertainty Propagation: PETALE in the CROCUS Reactor," Accepted in WONDER 2018 - 5th edition of the International Workshop On Nuclear Data Evaluation for Reactor Applications, 2018.

[5] OECD/NEA, Benchmark on Kinetic Parameters in the CROCUS Reactor, 4440. 2007, vol. 9, p. 94.

[6] Canberra Industries, "Genie TM 2000 Spectroscopy Software Operations,” pp. 61-97, 2006.

[7] R Venkataraman, F Bronson, V Atrashkevich, M Field, and B. M. Young, "Improved Detector Response Characterization Method in ISOCS and LabSOCS 1 . General Philosophy of ISOCS Detector Characterization 2 . Response Characterization Methodologies," vol. 264, no. Marc Vi, pp. 1-21, 2003.

[8] J. Leppänen, SERPENT- a Continuous-energy Monte Carlo Reactor Physics Burnup Calculation Code. 2015, p. 162.

[9] D. J. Siefman, G. Girardin, A. Rais, A. Pautz, and M. Hursin, "Full Core modeling techniques for research reactors with irregular geometries using Serpent and PARCS applied to the CROCUS reactor," Annals of Nuclear Energy, vol. 85, pp. 434-443, 2015.

[10] M. Reginatto, P. Goldhagen, and S. Neumann, "Spectrum unfolding, sensitivity analysis and propagation of uncertainties with the maximum entropy deconvolution code MAXED," Nuclear Instruments and Methods in Physics Research Section A: Accelerators, Spectrometers, Detectors and Associated Equipment, vol. 476, no. 1-2, pp. 242-246, 2002.

[11] S. Diamond and S. Boyd, "CVXPY: A Pythonembedded modeling language for convex optimization," Journal of Machine Learning Research, vol. 17, no. 83, pp. 1-5, 2016.

[12] A Santamarina, D Bernard, P Blaise, M Coste, A Courcelle, T. D. Huynh, C Jouanne, P Leconte, O Litaize, $\mathrm{S}$ Mengelle, G Noguère, J. M. Ruggiéri, O Sérot, J Tommasi, C Vaglio, J. F. Vidal, and Y Rugama, The JEFF-3.1.1 Nuclear Data Library, 22. 2009, pp. 1-62.

[13] R. Capote, K. Zolotarev, V. Pronyaev, and A. Trkov, "Updating and extending the irdf-2002 dosimetry library," 2012.

[14] D. Rochman, A. Koning, J. Sublet, M. Fleming, E. Bauge, S. Hilaire, P. Romain, B. Morillon, H. Duarte, S. Goriely, S. Van Der Marck, H. Sjöstrand, S. Pomp, N. DzysiGBR, O. Cabellos, H. FerroGBRhi, and A. Vasiliev, "The TENDL library: Hope, reality and future," EPJ Web of Conferences, vol. 146, 2017.

[15] M. Berger, J. Hubbell, S. Seltzer, J. Chang, J. Coursey, R. Sukumar, D. Zucker, and K. Olsen. (). Nist stan- 
dard reference database 8 , Com: Photon cross sections database.

[16] M. Tesinsky, C. Berglöf, T. Bäck, B. Martsynkevich, I. Serafimovich, V. Bournos, A. Khilmanovich, Y. Fokov, S. Korneev, H. Kiyavitskaya, and W. Gudowski, "Comparison of calculated and measured reaction rates obtained through foil activation in the subcritical dual spectrum facility YALINA-Booster," Annals of Nuclear Energy, vol. 38, pp. 1412-1417, 2011.

Table I: Activation characteristics for different foils

\begin{tabular}{|c|c|c|c|c|c|}
\hline Reaction & Abundance (\%) & Half-life (days) & $\begin{array}{l}\text { Threshold } \\
\text { Energy }(\mathrm{MeV})\end{array}$ & $\begin{array}{l}\text { Main } \\
(\mathrm{keV})\end{array}$ & $\begin{array}{l}\text { Activity } \\
\left(B q \cdot g^{-1} \cdot W^{-1}\right)\end{array}$ \\
\hline${ }^{46} \mathrm{Ti}(\mathrm{n}, \mathrm{p})^{46} \mathrm{Sc}$ & 8.25 & 83.8 & 1.6 & 8891020 & 0.237 \\
\hline${ }^{48} \mathrm{Ti}(\mathrm{n}, \mathrm{p}){ }^{48} \mathrm{Sc}$ & 73.72 & 1.82 & 3.3 & 9831037 & 2.73 \\
\hline${ }^{56} \mathrm{Fe}(\mathrm{n}, \mathrm{p})^{56} \mathrm{Mn}$ & 91.75 & 0.107 & 0.35 & 847 & $1.55 \times 10^{2}$ \\
\hline${ }^{58} \mathrm{Ni}(\mathrm{n}, \mathrm{p}){ }^{58} \mathrm{Co}$ & 68.08 & 70.8 & 0.4 & 811 & 20.0 \\
\hline${ }^{64} \mathrm{Ni}(\mathrm{n}, \gamma){ }^{65} \mathrm{Ni}$ & 0.927 & 0.105 & - & 1482 & $2.09 \times 10^{3}$ \\
\hline${ }^{63} \mathrm{Cu}(\mathrm{n}, \gamma){ }^{64} \mathrm{Cu}$ & 69.15 & 0.529 & - & 511 & $9.74 \times 10^{4}$ \\
\hline${ }^{65} \mathrm{Cu}(\mathrm{n}, \gamma){ }^{66} \mathrm{Cu}{ }^{1}$ & 30.85 & $3.54 \times 10^{-3}$ & - & 1345 & $3.89 \times 10^{5}$ \\
\hline${ }^{115} \operatorname{In}(\mathrm{n}, \gamma){ }^{116} \operatorname{In}$ & 95.72 & 0.0376 & - & 12941097 & $5.23 \times 10^{6}$ \\
\hline${ }^{115} \operatorname{In}\left(\mathrm{n}, \mathrm{n}^{\prime}\right)^{115 m} \mathrm{In}$ & 95.72 & 0.187 & 0.34 & 336 & $1.95 \times 10^{2}$ \\
\hline${ }^{197} \mathrm{Au}(\mathrm{n}, \gamma){ }^{198} \mathrm{Au}$ & 100 & 2.67 & - & 412 & $3.12 \times 10^{5}$ \\
\hline
\end{tabular}

${ }^{1}$ The activity corresponds to the activation after 1 hour of irradiation, when the foil is positioned in the central axis of CROCUS, at the middle height of the critical water level. 